\title{
A Study on Correlation Between Acanthosis Nigricans And Polycystic Ovarian Syndrome (PCOS) in Indian Adult Women Population
}

\author{
Bijay Khan ${ }^{1}$, Ranjan Basu ${ }^{1}$ \\ ${ }^{I}$ Assistant Professor, Dept. of Obstetrics and Gynecology; KPC Medical College, Kolkata
}

\begin{abstract}
Objective: To find the correlation of the clinical and biochemical parameters in Indian adult women with PCOS with and without $A N$.

Materials and Methods: 98 adult women (20-35years) with PCOS as per Rotterdam 2003 criteria were studied. Clinical parameters recorded - Body mass index (BMI), abdominal circumference (AC), blood pressure (BP), hirsutism score (modified Ferriman- Gallwey score $m F G \geq 6$ ), presence of acne and acanthosis (AN). Transabdominal ultrasonography (USG) was done in each case. Hormonal parameters studied - Serum total testosterone (TT) level, sex hormone binding globulin (SHBG) level. Free androgen index (FAI) was calculated. Metabolic parameters studied - fasting plasma glucose (FG) and insulin (FI) levels. Glucose: insulin (G:I) ratio and HOMA (homeostasis model assessment) were calculated. Studied women were divided into two groups Group A: With acanthosis nigricans - 30 cases. Group B: Without acanthosis nigricans- 68 cases.

Observations: Statistically significant differences between the means of groups with and without AN were found in the following variables - BMI ( $p$ value $=0.0001) ; A C$ ( $p$ value $=0.0001) ; S B P(p$ value $=0.0001) ; D B P(p$ value $=0.01) ; F G(p$ value $=0.12) ; S H B G(p$ value $=0.0001) ; F A I(p$ value $=0.0009) ; G I$ ratio $(p$ value $=0.0003)$ and HOMA ( $p$ value=0.0001). Bivariate positive Correlation with AN was observed between AC, BMI, SBP, $D B P$, Testosterone, FAI, and HOMA. Bivariate negative correlation with AN was observed with FG, SHBG and GI ratio. In terms of correlation coefficients, AN has the highest correlation with AC, followed by BMI and SHBG.

Conclusion: AN is a cutaneous manifestation of increased abdominal obesity in adult women having PCOS. Significance of AN as a clinical marker of increased metabolic risk in PCOS need further studies.
\end{abstract}

Keywords: Acanthosis nigricans (AN), adult, body mass index (BMI), polycystic ovary syndrome.

\section{Introduction}

Acanthosis Nigricans (AN) is a readily recognizable dermatosis characterized by velvety, papillomatous, brownish-black, hyperkeratotic plaques. It is seen on the intertriginous surfaces and neck ${ }^{1}$. It is a cutaneous manifestation of an underlying metabolic defect like insulin resistance (IR) or obesity ${ }^{2}$.

Presence of AN appears to be more of a sign of IR or medication reaction than distinct disease itself. Other pathological conditions rarely associated with AN are insulinoma and malignant diseases, especially adenocarcinoma of the stomach.

AN is also found in women with polycystic ovary syndrome (PCOS) because of its association with insulin resistance (IR) and hyperandrogenism (HA) ${ }^{3}$. It has been reported that between 5 and $33 \%$ of patients with PCOS have $\mathrm{AN}^{4,5}$.

Acanthosis is more common in PCOS patients with obesity. Hyperkeratosis and papillomatosis are the histological characteristics of AN. AN is a frequent occurrence in HA and diabetes mellitus ${ }^{6}$. In 1976, Kahn and colleagues ${ }^{7}$ found an association of HA and insulin resistance with AN.

There are very few studies which have reported the clinical, hormonal and metabolic parameters in PCOS with AN. In the present study various clinical and biochemical parameters (hormonal and metabolic) of adult Indian women having PCOS with and without AN are reported.

\section{Materials and Methods}

This comparative cross sectional observational study was done in KPC Medical College which is a tertiary care hospital, from March,2014 to April,2015.Permission was obtained from the ethics committee of the institute and all studied women agreed to the investigation protocol.

98 adult women (age 20-35 years) with PCOS were studied. These women had detailed clinical and hormonal evaluations for the diagnosis of PCOS according to the Rotterdam 2003 criteria ${ }^{8}$ with at least two of the following features: (i) oligo-ovulation or chronic anovulation, (ii) clinical and/or biochemical hyperandrogenism, and (iii) ultrasound appearance of polycystic ovaries. Secondary causes of 
hyperandrogenism, as per the Rotterdam criteria, were excluded by appropriate clinical and laboratory tests. Women with history of steroid or oral contraceptive drug intake in the preceding 3 months as well as previously diagnosed diabetes were also excluded from the study.

Oligo-ovulation and / or anovulation was characterized by oligomenorrhea (intermenstrual intervals of $\geq 35$ days) and amenorrhea (intervals $>3$ months). Clinical hyperandrogenism was defined as the presence of alopecia, or hirsutism (modified Ferriman-Gallwey score of $\geq 6$ ) and /or acne. Biochemical hyperandrogenism was considered if total testosterone level was more than $0.82 \mathrm{ng} / \mathrm{ml}$ (normal laboratory range $0.06-0.82 \mathrm{ng} / \mathrm{ml}$ ) or calculated free androgen index was more than $2.06^{9}$. Polycystic ovary on ultrasound (Transabdominal) was defined as the presence of at least one ovary $10 \mathrm{cc}$ or more in volume.

Fasting plasma glucose (FG) and Fasting plasma insulin (FI) levels were estimated after 12- hour overnight fasting for all subjects. Plasma glucose was measured by Glucose oxidase peroxidase method (Roche Diagnostics Gmbh, Mannheim, Germany) and was expressed in $\mathrm{mg} \%$ and plasma insulin level in $\mathrm{mcu} / \mathrm{ml}$. Glucose-insulin ratio (G:I) and homeostasis model assessment (HOMA) was calculated from FG and FI level. The method for assessing HOMA is FG $(\mathrm{mg} \%)$ x FI $(\mathrm{mcu} / \mathrm{ml}) / 405$. Serum total testosterone level (TT) was measured by using Electrochemiluminescence Immunoassay, Roche Lot. No. 181371- 01(Roche Diagnostics Gmbh, Mannheim,Germany) in ng/ml. Sex hormone binding globulin (SHBG) level was also measured (nmol/l) on the second or third day of progesterone induced bleeding. Free androgen index (FAI) was measured by the method $(\mathrm{TT} \times 100 \times 3.47) / \mathrm{SHBG}$.

A standard questionnaire was used to document length of menstrual cycles; personal, medical, and family history of diabetes; hypertension; obesity; and ischemic heart disease. Signs of androgen excess (hirsutism, acne, and alopecia), insulin resistance and presence of acanthosis were noted in the physical examination. Anthropometric measurements included abdominal circumference in centimeter as per internationally accepted guidelines (using a $1 \mathrm{~cm}$ wide measuring tape). Body mass index (BMI) $\left(\mathrm{kg} / \mathrm{m}^{2}\right) \mathrm{was}$ calculated in each case from height and weight measurements. Height was recorded to the nearest $0.5 \mathrm{~cm}$. Weight $(\mathrm{kg})$ was taken on a platform type (bathroom scale) machine, the accuracy of which was checked each time before weighing. Blood pressure was measured using a mercury sphygmomanometer, both systolic (SBP) and diastolic (DBP) BP was measured in $\mathrm{mm}$ of $\mathrm{Hg}$.

Trans-abdominal ultrasound was performed to study the morphology of ovaries. Ovarian volume measurements were carried out by measuring three perpendicular dimensions (volume for a prolate ellipsoid = $0.5 \times$ length $\times$ width $\times$ thickness). Follicle number was estimated both in longitudinal and antero-posterior crosssections of the ovaries. Secondary causes of hyperandrogenism like 21-hydroxylase deficiency, Cushing's syndrome, hypothyroidism, hyperprolactinemia, and androgen-secreting tumors were excluded by appropriate clinical and/or laboratory tests.

Assuming a confidence limit of $95 \%$, the calculated confidence interval was $10 \%$. For comparison of various clinical and biochemical parameters all parameters were tested for normality pre-test (using KS). The continuous variables were compared with $\mathrm{T}$ test and Chi-square test with Yates correction was also done as and when needed. To assess the degree of causality, logistic regression was done, (pseudo $\left.\mathrm{R}^{2}=0.3447\right)$, $\left(\mathrm{Prob}>\mathrm{chi}^{2}\right.$ $=0.0000$ ) predicting a good fit model, $\mathrm{p}$-value $<0.05$ was considered as significant.

\section{Result}

Table 1 shows the clinical parameters of the two groups of patients studied. Out of the 98 studied women, 30 were found to have Acanthosis Nigricans (Group A) (33.3\%) and 68 women did not have AN (Group B). There were significant differences in mean BMI ( $\mathrm{p}=0.0001)$, AC (0.0001), SBP ( $\mathrm{p}=0.0001)$, DBP $(\mathrm{p}=0.01)$ values between the two groups. On the other hand, $\mathrm{mFG}$ score for hirsutism showed no significant difference between the two groups $(\mathrm{p}=0.12)$.

Table 2 shows the biochemical parameters of the two groups of patients. SHBG $(\mathrm{p}=0.0001)$ and FAI $(\mathrm{p}=0.0009)$ values showed significant differences between the two groups whereas testosterone levels showed no significant difference $(p=0.24)$. Fasting plasma insulin $(p=0.0001)$, glucose: insulin ratio $(p=0.0003)$ and HOMA $(\mathrm{p}=0.0001)$ values showed significant differences between the two groups but no significant difference in glucose levels found $(\mathrm{p}=0.10)$.

By bivariate analysis it was found that $\mathrm{AN}$ had positive correlations with abdominal circumference, BMI, systolic and diastolic BP, serum testosterone level, FAI and HOMA, whereas it is found to be in negative correlation with fasting plasma glucose level, SHBG level and glucose-insulin ratio. In terms of correlation coefficient, AN had highest correlation with abdominal circumference, followed by BMI and SHBG level.

\section{Discussion}

The clinical importance of AN has been claimed to be due to its association with various metabolic and hormonal abnormalities such as obesity, diabetes, PCOS, dyslipidaemia, Cushing's syndrome, thyroid dysfunction etc ${ }^{10,11,12}$. 
The actual prevalence of AN in the population has not been assessed widely in India. Present study finds that $33.3 \%$ of adult PCOS women have AN. A hospital based study done in north India on selected group of diabetic patients and age matched controls had shown a very high prevalence of AN (62\% vs. $54 \%)^{13}$. But as this was not a community study and as most of the study subjects were obese, these results may not be representative of the population at large.

Many studies have shown that fasting insulin level can be considered a good indicator of insulin resistance ${ }^{14,15,16}$. Present study has demonstrated higher mean fasting insulin values among subjects with AN denoting higher insulin resistance among them compared to subjects without AN and are comparable to the results of a similar study conducted in Mumbai, India ${ }^{17}$.

Menon et al ${ }^{18}$ in their study done among south Indian population reported that obesity might be a contributory factor for the high insulin level. They found that though mean insulin level were higher among all BMI categories with AN, it was statistically significant only among the obese group. This suggests that AN is more significantly associated with insulin resistance among the obese population than normal or overweight subjects. Our study also shows that AN in adult woman with PCOS is another clinical marker of obesity. We have found that presence of $\mathrm{AN}$ is not always an indicator of insulin resistance, as detected by the fasting G: I ratio - rather obesity is a more consistent feature with AN. Panidis et al ${ }^{19}$ had stated in their study that insulin resistance is a necessary, but not the only factor leading to the development of AN in patients with PCOS.

Our study has found that AN is strongly correlated with abdominal circumference (highest correlation) followed by BMI and negative correlation with G: I ratio. This suggests that presence of AN should direct the clinician to give advices to patients in reducing abdominal circumference through changes in lifestyle pattern.

\section{Conclusion}

This study signifies that presence of AN has a significant role as an indicator of central obesity and consequent metabolic disorders. In a clinical setting presence of AN should direct the clinician to suspect underlying metabolic problems associated with PCOS and take appropriate preventive measures for future metabolic problems in them.

\section{Acknowledgement}

The author is extremely grateful to Mr.S.M.Bhattacharjee M.Sc for carrying out the statistical analysis and inferences.

[1]. J P Burke et al: A Quantitative Scale of Acanthosis Nigricans

[2]. Diabetes Care 1999: 22 (10),1655- 9

[3]. Barbieri RL, Ryan KJ. Hyperandrogenism, insulin resistance, and acanthosis nigricans syndrome: A common endocrinopathy with distinct pathophysiologic features. Am J Obstet Gynecol 1983; 147:90-101.

[4]. 3. Guzick DS. Polycystic ovary syndrome. Obstet Gynecol 2004; 103:181-193.

[5]. Rendon MI, Cruz PD, Jr, Sontheimer RD, Bergstresser PR. Acanthosis nigricans: A cutaneous marker of tissue resistance to insulin. J Am Acad Dermatol 1989; 21: 461-9.

[6]. Maitra SK, Rowland Payne CM. The obesity syndrome and acanthosis nigricans. Acanthosis nigricans is a common cosmetic problem providing epidemiological clues to the obesity syndrome, the insulin-resistance syndrome, the thrifty metabolism, dyslipidaemia, hypertension and diabetes mellitus type II. J Cosmet Dermatol2004;3: 202-10.

[7]. Kierland RR. Acanthosis nigricans; an analysis of data in 22 cases and a study of its frequency in necropsy material. $\mathrm{J}$ Invest Dermatol 1947; 9:299-305.

[8]. Kahn CR, Flier JS, Bar RS, Archer JA, Gorden P, Martin MM, et al. The syndromes of insulin resistance and acanthosis nigricans. Insulin-receptor disorders in man. N Engl J Med 1976; 294:739-45.

[9]. Rotterdam ESHRE/ASRM-Sponsored PCOS Consensus Workshop Group.

[10]. Revised 2003 consensus on diagnostic criteria and long-term health risks related to polycystic ovary syndrome. Fertil Steril 2004:81(1):19-25.

[11]. Bhattacharya SM. Hyperandrogenism in oligomenorrhoea with minimal or nil 'unwanted hair growth' . Gynaecol Endocrinol 2009: July: 25(7): 423-6

[12]. Araujo L.M., Port M.V., Netto E.M., Ursich M.J.: Association of Acanthosis Nigricans with race and metabolic disturbances in obese women. Braz J Med Biol Res 2002: 35: 59-64.

[13]. Ten S., Maclaren N.: Insulin resistance syndrome in children. J Clin Endocrinol Metab 2004: 89: 2526- 2539.

[14]. Litonjua P., Pinero-Plona A., Aviles-Santa L., Raskin P.: Prevalence of Acanthosis Nigricans in newly diagnosed type 2 diabetes. Endocr Pract 2004;10: 101-106.

[15]. Grandhe N.P., Bhansali A., Dogra S., Kumar B.: Acanthosis Nigricans: relation with type 2 diabetes mellitus, anthropometric variables and body mass index in Indians. Postgrad Med J 2005; 81: 541-544.

[16]. Ten S., Maclaren N.: Insulin resistance syndrome in children. J Clin Endocrinol Metab 2004; 89: $2526-2539$.

[17]. Kim S.H., Abbasi F., Reaven G.: Impact of degree of obesity on surrogate estimates of insulin resistance. Diabetes Care 2004; 27: 1998-2002.

[18]. Yamazaki H., Ito S., Yoshida H.: Acanthosis Nigricans is a reliable cutaneous marker of insulin resistance in obese Japanese children. Pediatr Int 2003; 45: 701-705.

[19]. Varthakavi P.K., Patel K.L., Wadhwa S.L., Khopkar U., Sen Guptha R.A., Merchant P.C., et al: A study of insulin resistance in subjects with Acanthosis Nigricans. J Assoc Physicians India 2001; 49. 705- 712. 
A study on correlation between Acanthosis Nigricans and Polycystic Ovarian Syndrome(PCOS) in Indian adult..

[20]. Usha Menon V, Vinod Kumar K, Gilchrist A, Sundaram KR, Jayakumar RV, Nair V, Kumar H. Acanthosis Nigricans and Insulin levels in a south Indian population- (ADEPS paper 2). Obesity Research \& Clinical Practice. 2008: 2: 43-50.

[21]. Panidis D, Skiadopoulos S, Rousso D, Ioannides D, Panidou E. Association of acanthosis nigricans with insulin resistance in patients with polycystic ovary syndrome. Br J Dermatol 1995;132:936-41.

Table 1: Clinical parameters in the two groups of patients Data are mean (Standard deviation)

\begin{tabular}{|l|l|l|l|}
\hline Parameters & $\begin{array}{l}\text { AN absent } \\
(\mathrm{n}=68)\end{array}$ & $\begin{array}{l}\text { AN present } \\
(\mathrm{n}=30)\end{array}$ & $\mathrm{p}$ value \\
\hline $\mathrm{BMI}\left(\mathrm{kg} / \mathrm{m}^{2}\right)$ & $24.31(3.22)$ & $28.8(4.31)$ & 0.0001 \\
\hline $\mathrm{AC}(\mathrm{cm})$ & $76.72(2.8)$ & $88.91(3.8)$ & 0.0001 \\
\hline $\mathrm{mFG}$ score & $6.4(5.6)$ & $4.5(5.1)$ & 0.12 \\
\hline SBP $(\mathrm{mm}$ of $\mathrm{Hg})$ & $122.12(11.81)$ & $126.62(13.9)$ & 0.0001 \\
\hline DBP $(\mathrm{mm}$ of $\mathrm{Hg})$ & $80.13(10.4)$ & $81.82(10.1)$ & 0.01 \\
\hline
\end{tabular}

Table 2: Biochemical parameters of the two group.Data are mean (Standard deviation)

\begin{tabular}{|l|l|l|l|}
\hline Parameters & AN absent $(\mathrm{n}=68)$ & AN present $(\mathrm{n}=30)$ & $\mathrm{p}$ value \\
\hline Testosterone $(\mathrm{ng} / \mathrm{ml})$ & $0.47(0.3)$ & $0.58(0.3)$ & 0.24 \\
\hline SHBG $(\mathrm{nmol} / \mathrm{l})$ & $34.41(23.4)$ & $22.73(13.1)$ & 0.0001 \\
\hline FAI & $7.13(8.6)$ & $10.82(7.3)$ & 0.0009 \\
\hline Fasting glucose $(\mathrm{mg} \%)$ & $90.5(8.08)$ & $94.2(6.28)$ & 0.10 \\
\hline $\begin{array}{l}\text { Fasting Insulin } \\
\text { (mcu / ml) }\end{array}$ & $11.21(7.9)$ & $22.13(19.1)$ & 0.0001 \\
\hline Glucose: Insulin & $11.73(7.5)$ & $8.81(15.6)$ & 0.0003 \\
\hline HOMA & $2.5(1.8)$ & $5.3(4.9)$ & 0.0001 \\
\hline
\end{tabular}

